\title{
Daclatasvir Plus Asunaprevir for the Treatment of Patients with Hepatitis C Virus Genotype 1b Infection: Real-World Efficacy, Changes in Liver Stiffness and Fibrosis Markers, and Safety
}

\author{
Hye Won Lee ${ }^{1,2,3}$, Se Rim Oh ${ }^{1}$, Dong Yun Kim ${ }^{1}$, Yechan Jeong ${ }^{1,4}$, Seungtaek Kim ${ }^{1,2,3}$, Beom Kyung Kim ${ }^{1,2,3}$, \\ Seung Up Kim ${ }^{1,2,3}$, Do Young Kim ${ }^{1,2,3}$, Sang Hoon Ahn ${ }^{1,2,3}$, Kwang-Hyub Han ${ }^{1,2,3}$, and Jun Yong Park ${ }^{1,2,3}$ \\ ${ }^{1}$ Department of Internal Medicine and ${ }^{2}$ Institute of Gastroenterology, Yonsei University College of Medicine, ${ }^{3}$ Yonsei Liver Center, Severance \\ Hospital, Yonsei University College of Medicine, and ${ }^{4}$ Brain Korea 21 PLUS Project for Medical Science College of Medicine, Seoul, Korea
}

Background/Aims: The treatment with daclatasvir plus asunaprevir (DCV+ASV) is associated with potent antiviral effects in patients with genotype $1 \mathrm{~b}$ hepatitis $\mathrm{C}$ virus (HCV) infection. We investigated the real-world efficacy, changes in liver stiffness and noninvasive fibrosis markers, and the safety of DCV+ASV treatment in Korean patients. Methods: In total, 363 patients with chronic hepatitis $C$ were treated with DCV+ASV between August 2015 and January 2017. Finally, we analyzed the data of 270 patients who were monitored for at least 12 weeks after the end of treatment. Results: The mean age was 60.7 years, and females predominated (60.4\%). Most patients (64.8\%) were treatment-naïve, and 56 patients $(20.7 \%)$ had cirrhosis. Two hundred fifty-seven (95.2\%) and 251 (93.0\%) patients achieved end-of-treatment responses and sustained virological responses at 12 weeks posttreatment (SVR12), respectively. The SVR12 rates were higher in patients who were $<65$ years of age, males, without cirrhosis and had lower HCV RNA levels. All LS values and fibrosis-4 and aspartate aminotransferase-to-platelet ratio index values declined from baseline to the time of assessment of SVR12. Conclusions: The DCV+ASV therapy resulted in a high SVR12 and improved liver fibrosis; the treatment was well tolerated in patients with genotype $1 \mathrm{~b} \mathrm{HCV}$ infections. (Gut Liver 2018;12:324-330)

Key Words: Daclatasvir; Asunaprevir; Hepatitis C; Genotype $1 \mathrm{~b}$; Fibrosis

\section{INTRODUCTION}

Approximately 180 million subjects are chronically infected with hepatitis $\mathrm{C}$ virus (HCV) worldwide. ${ }^{1,2} \mathrm{HCV}$ infection is one of the leading causes of chronic liver disease, cirrhosis and hepatocellular carcinoma (HCC). ${ }^{3,4} \mathrm{HCV}$ is a single-stranded positive RNA virus with seven genotypes. Genotype $1 \mathrm{~b}$ is the most predominant subtype in Asia, constituting 45\% to 55\% of all genotypes infecting patients in Korea and Taiwan. ${ }^{5,6}$ HCV treatments have evolved rapidly with the development of directacting antiviral (DAA) therapies.

In HCV patients, DAA therapies are associated with higher sustained virological response (SVR) rates, reduced treatment durations, and minimal side-effects. ${ }^{7}$ DAAs target principally the HCV nonstructural protein (NS) 3/4A serine protease, the NS5A protein, and the NS5B polymerase. ${ }^{8}$ Daclatasvir (DCV; an NS5A replication complex inhibitor) plus asunaprevir (ASV; an NS3 protease inhibitor) was recently approved in Korea as the firstline DAA treatment for patients chronically infected by HCV genotype $1 \mathrm{~b} .^{9,10}$ In a pooled analysis, a DCV+ASV regimen was effective and well-tolerated in patients with genotype $1 \mathrm{~b} \mathrm{HCV}$ infection, regardless of the presence of cirrhosis. ${ }^{11}$ However, real-world data often differ from those of clinical trials because clinical trials impose several strict inclusion and exclusion criteria. Thus, the collection of real-life data is essential to confirm treatment efficacy and safety in clinical settings.

DCV is a potent pan-genotypic NS5A inhibitor exhibiting antiviral activity across HCV genotypes 1 to 6 in vitro. ASV is an NS3 protease inhibitor active against genotypes 1, 4, 5, and 6 in vitro. ${ }^{12}$ Dual therapy with DCV+ASV for 24 weeks, without the use of pegylated interferon/ribavirin (PegIFN/RBV), afforded

Correspondence to: Jun Yong Park

Department of Internal Medicine, Yonsei University College of Medicine, 50-1 Yonsei-ro, Seodaemun-gu, Seoul 03722, Korea

Tel: +82-2-2228-1988, Fax: +82-2-393-6884, E-mail: drpjy@yuhs.ac

Received on July 7, 2017. Revised on September 20, 2017. Accepted on September 20, 2017. Published online February 8, 2018 pISSN 1976-2283 eISSN 2005-1212 https://doi.org/10.5009/gnl17298

(c) This is an Open Access article distributed under the terms of the Creative Commons Attribution Non-Commercial License (http://creativecommons.org/licenses/by-nc/4.0) which permits unrestricted non-commercial use, distribution, and reproduction in any medium, provided the original work is properly cited. 
high SVR rates in treatment-naïve patients and those who were ineligible/intolerant (87\%) or nonresponsive (81\%) to PegIFN/ RBV treatment, provided that they lacked resistance-associated substitutions (RAS). ${ }^{13-15}$ Moreover, DCV+ASV treatment was associated with favorable SVR rates and low levels of adverse events, even in patients with compensated cirrhosis. ${ }^{16,17}$

Viral eradication with IFN-treatment of chronic hepatitis C (CHC) liver fibrosis and reduces the incidences of HCC and liverrelated mortality. However, any long-term benefit afforded by viral eradication in $\mathrm{HCV}$-infected patients using IFN-free DAA combination therapy, remains unknown. ${ }^{18-20}$ A recent study founded that improvement of fibrosis was important in $\mathrm{CHC}$ patients who treated with sofosbuvir-based regimen. ${ }^{21}$ Although the efficacy and changes in fibrosis have been assessed in clinical trials, few real-world data are available. ${ }^{17,22}$ Therefore, we investigated the real-world efficacy, changes in liver stiffness (LS) values and the fibrosis markers, and safety in Korean patients with genotype $1 \mathrm{~b} \mathrm{HCV}$ infection.

\section{MATERIALS AND METHODS}

\section{Patients}

A total of 363 patients chronically infected with HCV genotype $1 \mathrm{~b}$ started 24 weeks treatment with DCV+ASV at Severance Hospital, Yonsei University College of Medicine, Seoul, Korea between August 2015 and January 2017. The doses were DCV $60 \mathrm{mg}$ once daily and ASV $100 \mathrm{mg}$ twice daily. All data were prospectively collected using an established protocol and were followed up for 12 weeks posttreatment.

The inclusion criteria were age $\geq 20$ years, CHC infection with genotype $1 \mathrm{~b}$ only, and a detectable HCV RNA titer without any RAS (Fig. 1). Patients who were nonresponsive to, ineligible for, or intolerant of previous treatment, and those who had relapsed, were included. The exclusion criteria were: (1) hepatic decompensation evident at the time of enrollment or a prior history of decompensation; (2) co-infection with other HCV genotypes or hepatitis B virus; (3) RAS positive at L31 or Y93 ( $\mathrm{n}=36)$; (4) HCC evident at enrollment $(n=53)$; and (5) loss to follow-up $(n=4)$. Our analyses of efficacy and safety included all patients who received at least one dose of medication.
Written informed consent was obtained from all participants. The study was approved by the Institutional Review Board of Severance Hospital and conformed to the ethical guidelines of the 1975 Helsinki Declaration (IRB Number: 4-2015-0701).

\section{Clinical and laboratory evaluation}

The RASs in the HCV NS5A and HCV NS3 regions were analyzed by Sanger direct-sequencing methods. Clinical data including age, sex, HCV genotype, HCV RNA titer, comorbidities, prior HCV treatment history, and the presence of cirrhosis was assessed at baseline. Clinical assessment was performed at weeks 4, 12, 24 and 36. At each visit, a complete blood cell count, routine blood chemistry tests, and side effects were checked. HCV RNA level were checked at baseline; at 4, 12, and 24 weeks of treatment; and at 12 weeks after the end of treatment (EOT). Serum HCV RNA levels were quantified using a commercial polymerase chain reaction assay (Amplicor HCV; Roche Diagnostics, Seoul, Korea), which has a lower limit of quantification of $15 \mathrm{IU} / \mathrm{mL}$. Serum alanine aminotransferase (ALT) levels were measured using a standard laboratory procedure with the upper limit of normal set to $33 \mathrm{IU} / \mathrm{mL}$ in males and $25 \mathrm{IU} / \mathrm{mL}$ in females. ${ }^{23}$ During the follow-up period, all patients underwent periodic ultrasonographic and laboratory work-ups, including measurement of $\alpha$-fetoprotein levels every 3 or 6 months to screen for HCC and other portal hypertension-related complications.

Cirrhosis was diagnosed by liver biopsy (METAVIR score 4 or Ishak score 5, 6), by transient elastography (TE) (LS value $>12.5$ kilopascal $[\mathrm{kPa}]$ ), or by measurement of laboratory biomarkers (aspartate aminotransferase to platelet ratio index [APRI] $>2.0$ and fibrosis-4 [FIB-4] score $>3.25 .^{24,25}$

\section{Changes in fibrosis assessed by noninvasive markers}

To assess the effect of DCV+ASV treatment, liver fibrosis markers, including the APRI and FIB-4 scores, were measured in all patients at baseline, at the EOT, and at 12 weeks after treatment. An APRI $>2.0$ predicts cirrhosis and a FIB-4 $>3.25$ predicts advanced fibrosis with a positive predictive value of $65 \%{ }^{26,27}$ LS values were assessed by TE both at baseline and SVR12. TE examinations were performed by a single experi-
363 Patients with HCV genotype $1 \mathrm{~b}$ infection receiving daclatasvir plus asunaprevir
- History of hepatic decompensation

- Co-infection with other HCV genotypes or HBV

- RAS positive at L31 or Y93

- HCC at enrollment

- Loss to follow-up loss
Fig. 1. Recruitment algorithm $\mathrm{HCV}$, hepatitis C virus; HBV, hepatitis B virus; RAS, resistance-associated substitutions; HCC, hepatocellular carcinoma. 
enced technician ( $>20,000$ examinations) blinded to patient data. The results were expressed in kilopascal and the median value of successful measurements was selected as representative LS value. The interquartile range (IQR) was defined as an index of the intrinsic variability of LS values corresponding to the interval of LS results containing 50\% of the valid measurements between the 25th and 75th percentiles. Only LS values derived from $\geq 10$ valid shots, a success rate $\geq 60 \%$, and an IQR to median value ratio $<0.3$ were considered reliable, and used in statistical analysis. $^{28}$

\section{Assessment of treatment efficacy and safety}

The primary endpoint was to evaluate SVR12, defined as undetectable HCV RNA at 12 weeks posttreatment. The second endpoint was to evaluate the changes in liver fibrosis assessed by noninvasive methods before and after DCV+ASV treatment. Treatment responses were defined as follows: relapse; an end of treatment response (ETR), defined as undetectable HCV RNA at the end of treatment; virological breakthrough (VBT); ALT normalization. Adverse events were assessed at each visit.

\section{Statistical analysis}

Data are expressed as mean \pm standard deviation (SD), median (with range), or numbers (with percentages), as appropriate. Continuous and categorical variables were compared using Student t-test (or the Mann-Whitney U-test, as appropriate); and the chi-square test (or Fisher exact test, as appropriate), respectively. All statistical analyses were performed using SPSS version 20.0 (IBM Corp., Armonk, NY, USA). A p-value <0.05 was considered statistically significant.

\section{RESULTS}

\section{Baseline characteristics}

A total of 270 patients followed up for at least 12 weeks after treatment were finally analyzed. Their baseline demographic characteristics are summarized in Table 1 . The mean patient age was 60.7 years (SD, 12.4 years), and females ( $n=163,60.4 \%)$ predominant. Treatment-naïve patients $(n=175,64.8 \%)$ were in the majority. The mean baseline HCV RNA level was $5.9 \log _{10}$

Table 1. Baseline Characteristics of the Study Population $(n=270)$

\begin{tabular}{lc}
\hline \multicolumn{1}{c}{ Variable } & Value \\
\hline Age, yr & $60.7 \pm 12.4$ \\
$<65$ & $156(57.8)$ \\
$\geq 65$ & $114(42.2)$ \\
Female sex & $163(60.4)$ \\
Cirrhosis & $56(20.7)$ \\
Body mass index, kg/m ${ }^{2}$ & $23.5 \pm 3.2$ \\
Prior HCV therapy & \\
Treatment-naïve & $175(64.8)$ \\
$\quad$ Non-responders & $37(13.7)$ \\
IFN/RBV ineligible/intolerant & $31(11.5)$ \\
$\quad$ Relapsers & $27(10.0)$ \\
HCV RNA, log10 IU/mL & $5.9 \pm 0.9$ \\
Aspartate aminotransferase, IU/L & $54.3 \pm 39.1$ \\
Alanine aminotransferase, IU/L & $46.6 \pm 39.1$ \\
Platelet count, $10^{3} / \mu \mathrm{L}$ & $164.8 \pm 65.4$ \\
Total cholesterol, mg/dL & $167.3 \pm 31.3$ \\
$\alpha-$ Fetoprotein , ng/mL & $5.9 \pm 0.8$ \\
Liver stiffness values, $\mathrm{kPa}$ & $13.2 \pm 12.6$ \\
CAP, dB/m & $228.6 \pm 38.1$ \\
APRI score & $1.13 \pm 1.25$ \\
FIB-4 score & $4.39 \pm 4.66$ \\
\hline D are presente &
\end{tabular}

Data are presented as mean \pm SD or number (\%).

$\mathrm{HCV}$, hepatitis C virus; IFN, interferon; RBV, ribavirin; CAP, controlled attenuation parameter; APRI, aspartate aminotransferase to platelet ratio index; FIB-4, fibrosis-4.
A

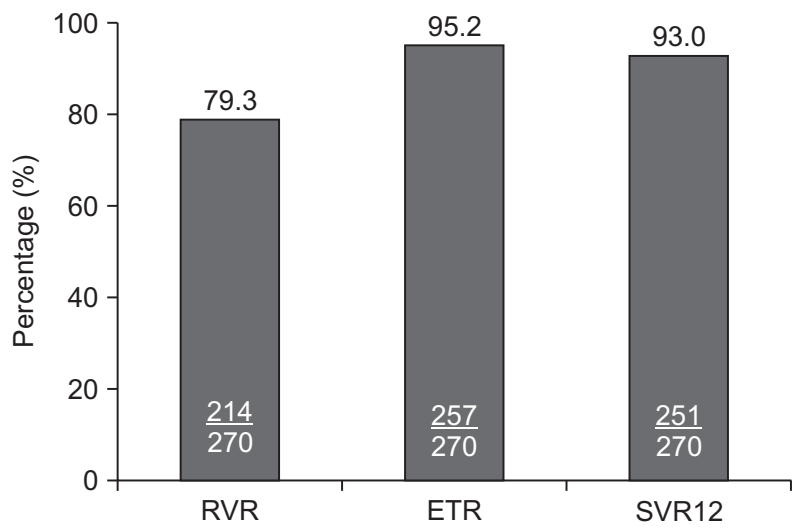

B

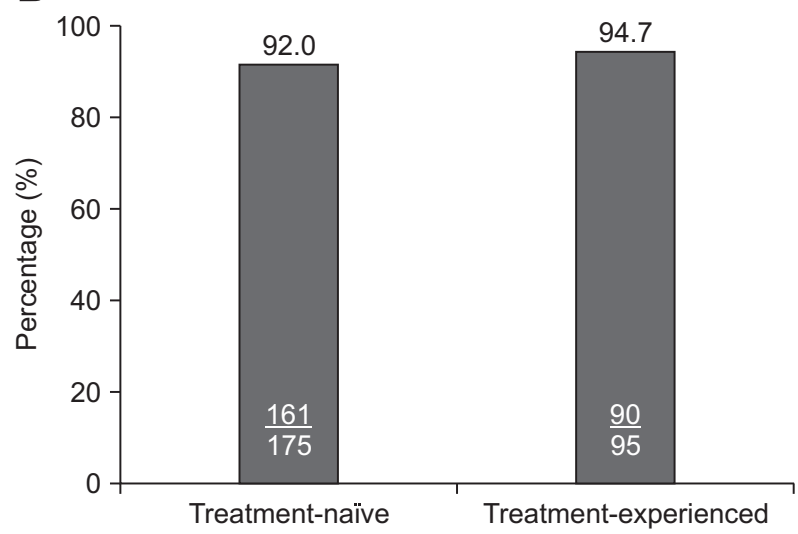

Fig. 2. (A) Treatment responses at baseline, 4 weeks, end of treatment, and 12 weeks after daclatasvir+asunaprevir treatment in the entire study population; (B) SVR12 rates by prior treatment experience.

RVR, rapid virologic response; ETR, end of treatment response; SVR12, sustained virological response at 12 weeks posttreatment. 
IU/mL (SD, $0.9 \log _{10} \mathrm{IU} / \mathrm{mL}$ ). Of all patients, 56 (20.7\%) had cirrhosis. The mean LS value was $13.2 \mathrm{kPa}(\mathrm{SD}, 12.6 \mathrm{kPa})$ at baseline.

\section{Virological response}

Of all patients, 214 patients (79.3\%) achieved rapid virologic response defined as undetectable HCV RNA at week 4 of therapy. Two hundred fifty-seven (95.2\%) and 251 (93.0\%) patients achieved ETR and SVR12, respectively (Fig. 2A). The SVR12 rates by prior treatment history were 92.0\% (161/175) in treatment-naïve patients and 94.7\% (90/95) in those with treatment-experienced (35/37 in non-responders, 30/31 in IFN/ RBV ineligible/intolerant patients, and 25/27 in relapsers) (Fig. 2B). The SVR12 rates were higher in patients $<65$ years than in those $\geq 65$ years of age $(91.9 \%, 136 / 148$ vs $90.4 \%, 103 / 114)$ (Fig. 3). The SVR rate was higher in males $(94.4 \%, 101 / 107)$ than females $(92.0 \%, 150 / 163)$. SVR12 was achieved both in patients with cirrhosis $(87.5 \%, 49 / 56)$ and those without cirrhosis $(94.4 \%, 202 / 214)$. When subdivided by the HBV RNA level,



Fig. 3. SVR 12 rates following 24 weeks of treatment with daclatasvir+asunaprevir according to age, sex, cirrhosis status, and baseline hepatitis $\mathrm{C}$ virus (HCV) RNA level.
95.2\% (119/125) of patients with levels $\geq 6 \log _{10} \mathrm{IU} / \mathrm{mL}$ and $91.0 \%(132 / 145)$ of patients with levels $<6 \log _{10} \mathrm{IU} / \mathrm{mL}$ achieved SVR12. The SVR rate was 92.3\% (12/13) in patients with estimated glomerular filtration rates (eGFR) $<50 \mathrm{~mL} / \mathrm{min} / 1.73 \mathrm{~m}^{2}$ and 93.0\% (239/257) in those with eGFR $\geq 50 \mathrm{~mL} / \mathrm{min} / 1.73 \mathrm{~m}^{2}$. In patients with and without diabetes mellitus, 93.5\% (29/31) and 92.9\% (222/239) of patients achieved SVR12, respectively.

\section{Safety and virological failure}

Of patients who had no HCC at baseline and were RAS negative, 16 patients discontinued DCV+ASV treatment prior to 24 weeks because of the development of adverse events $(n=5)$, treatment failure $(n=3)$, and VBT $(n=8)$. One patient discontinued DCV+ASV treatment at week 2 because of gastrointestinal problem. Another developed a skin rash and stopped treatment at week 12. One 63-year-old female patient exhibited mild creatinine elevation (from 1.3 to $2.3 \mathrm{mg} / \mathrm{dL}$ at week 4) and refused to continue treatment. One 71-year-old female patient stopped treatment at week 4 because of general weakness. One patient discontinued treatment because of uncontrolled ascites at week 20. Among five patients who experienced adverse events, four (80\%) had cirrhosis at baseline. Three patients experienced ETR, but recurred at 12 weeks after treatment completion.

$\mathrm{DCV}+\mathrm{ASV}$ treatment was relatively safe, but one patient experienced drug-drug interactions. She visited the hospital at 24 weeks and we confirmed ETR. However, she was admitted for treatment of severe hepatitis (ALT >5-fold the upper limit of normal), even though her HCV RNA status was negative. The hepatitis was caused by itraconazole for treatment 1 week in duration; the drug had been prescribed in another clinic to treat vaginitis. After conservative care, she was discharged with improved liver enzyme levels and achieved both an ETR and SVR12. Two patients who achieved SVR12 developed HCC. Both had cirrhosis at baseline and developed HCC approximately 3 months after the end of treatment.

\section{Changes in liver fibrosis}

The LS values obtained by TE, showed that $49.0 \%$ of patients had non-significant fibrosis (grade $<$ F2), 10.2\% mild to

Table 2. Changes in Noninvasive Fibrosis Markers at Baseline and SVR12

\begin{tabular}{|c|c|c|c|c|c|c|c|c|c|c|}
\hline & \multirow{2}{*}{$\begin{array}{c}\text { No. of } \\
\text { patients }\end{array}$} & \multicolumn{3}{|c|}{ Liver stiffness values } & \multicolumn{3}{|c|}{ FIB-4 } & \multicolumn{3}{|c|}{ APRI } \\
\hline & & Baseline & SVR12 & $\mathrm{p}$-value & Baseline & SVR12 & p-value & Baseline & SVR12 & $\mathrm{p}$-value \\
\hline Total & $270(100)$ & $13.2 \pm 12.8$ & $10.5 \pm 8.8$ & & $4.57 \pm 4.78$ & $3.03 \pm 3.08$ & & $1.40 \pm 1.36$ & $0.60 \pm 0.54$ & \\
\hline Cirrhosis & $56(20.7)$ & $25.5 \pm 17.5$ & $19.8 \pm 11.4$ & $<0.001$ & $8.76 \pm 6.23$ & $5.19 \pm 4.98$ & $<0.001$ & $2.39 \pm 1.10$ & $1.13 \pm 0.70$ & $<0.001$ \\
\hline No cirrhosis & $214(79.3)$ & $8.6 \pm 6.0$ & $6.7 \pm 3.2$ & $<0.001$ & $2.87 \pm 2.55$ & $2.34 \pm 1.66$ & 0.001 & $0.97 \pm 1.23$ & $0.38 \pm 0.23$ & $<0.001$ \\
\hline Treatment experienced & $95(35.2)$ & $17.9 \pm 16.4$ & $14.1 \pm 11.3$ & $<0.001$ & $6.24 \pm 6.16$ & $3.98 \pm 4.34$ & 0.003 & $1.91 \pm 1.75$ & $0.76 \pm 0.70$ & $<0.001$ \\
\hline
\end{tabular}

Data are presented as number $(\%)$ or mean \pm SD.

SVR, sustained virological response; FIB-4, fibrosis-4; APRI, aspartate aminotransferase to platelet ratio index. 

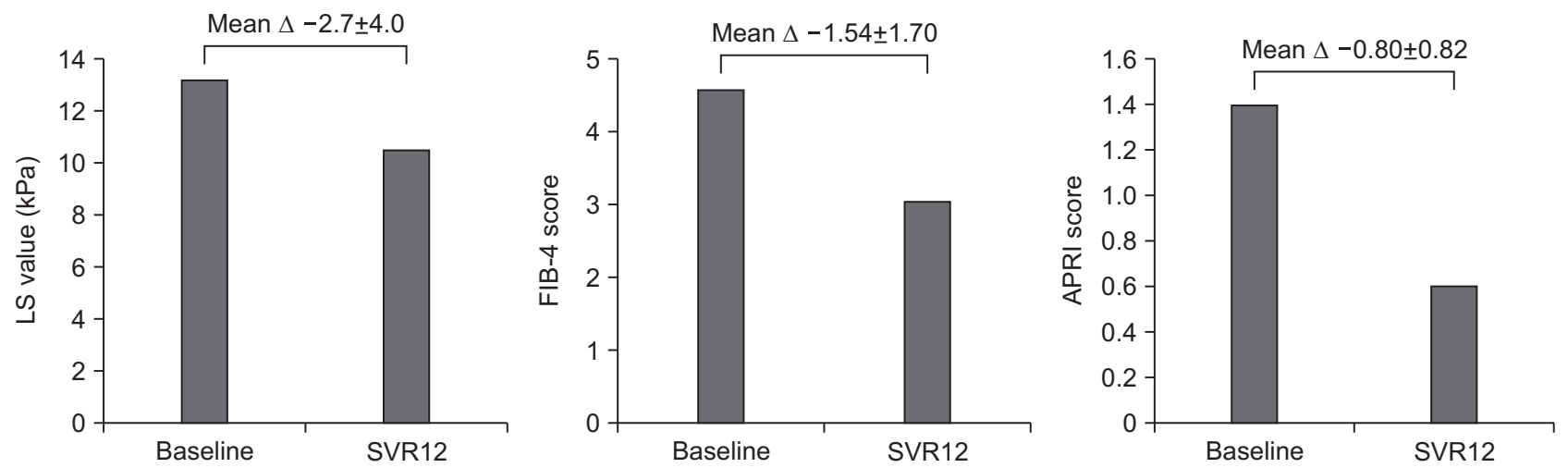

Fig. 4. Mean changes from baseline to 12 weeks posttreatment in noninvasive markers of liver fibrosis. LS, liver stiffness; SVR12, sustained virological response at 12 weeks posttreatment; FIB-4, fibrosis-4; APRI, aspartate aminotransferase to platelet ratio index.

significant fibrosis (F2-F3), 10.2\% advanced fibrosis (F3-F4) and $30.6 \%$ cirrhosis (F4). The FIB-4 score was $4.39 \pm 4.66$ and the mean APRI 1.13 \pm 1.30 . All patients exhibited declines in the levels of noninvasive fibrosis markers at SVR12 compared with baseline. Significant decline were also evident in the LS, FIB-4, and APRI values from baseline to SVR12 regardless of cirrhosis status or any prior history of treatment (Table 2). The overall LS values by TE decreased significantly from $13.2 \mathrm{kPa}$ at baseline to $10.5 \mathrm{kPa}$ at SVR12 $(\mathrm{p}<0.001)$. The overall APRI and FIB4 scores also decreased significantly (Fig. 4). The mean APRI score prior to therapy was 1.40 and that after therapy was 0.60 $(\mathrm{p}<0.001)$. The mean FIB-4 value was 4.57 prior to treatment and 3.03 at 12 weeks posttreatment.

\section{DISCUSSION}

We investigated the real-world efficacy of DCV+ASV treatment in patients infected with genotype $1 \mathrm{~b}$ HCV. DCV+ASV treatment was approved in 2015; and our center has treated the greatest number of patients and has the most extensive collection of long-term data as a single institution in Korea. HCV infection is the second most common cause of chronic liver disease in Korea. HCV prevalence is especially highest among older patients, with rapid disease progression. Of all HCV patients, 2\% were $\geq 70$ years of age, and were predominantly female. HCV genotype $1 \mathrm{~b}$ is the most prevalent subtype in Korea; the prevalence ranges from $45 \%$ to $59 \%{ }^{29,30}$

ETR was achieved in 95.2\% and SVR12 was achieved in 93.0\% of all patients. Thus, Korean patients with HCV genotype $1 \mathrm{~b}$ responded well to treatment regardless of age, sex, cirrhosis status, or HCV RNA levels. In previous studies, DCV+ASV treatment was associated with SVR12 in 90\% of treatment-naïve patients, $82 \%$ of non-responders, and $82 \%$ of ineligible/intolerant patients. ${ }^{11}$ Our results were similar; $92.0 \%$ of treatment-naïve patients achieved SVR12. Ninety-four point six percent (35/37) patients of non-responders, 96.0\% (30/31) of ineligible/intoler- ant patients, and 92.6\% (25/27) patients of relapsers showed SVR12.

We treated 36 (9.9\%, 36/363) patients with NS5A-Y93 (n=28) mutations and/or NS5A-L31 mutations ( $\mathrm{n}=9$ ). Nineteen patients (52.8\%) achieved SVR12. Three patients exhibited VBT and two were non-responders. The SVR rate of RAS-positive patients was slightly higher than that observed in previous clinical trials. We sequenced the viral genomes of five patients who failed $\mathrm{DCV}+\mathrm{ASV}$ treatment. The well-known Y93H mutation of NS5A was observed in four. Substitution mutations at Leu31 (Val or Met) of NS5A were also observed in three out of the four Y93Hcontaining viruses. However, one genome had no substitution mutation at Leu31 and Tyr93 having, rather, a deletion mutation at Pro32.

Overall, 87.5\% of cirrhotic patients achieved SVR12 (87.5\% in cirrhosis vs $94.4 \%$ in non-cirrhosis, $\mathrm{p}=0.908$ ). This low treatment outcome is probably due to the low number of patients with cirrhosis $(n=56)$ compared with non-cirrhosis $(n=214)$, so a larger number of cirrhotic patients is needed. The concentration of ASV after treatment commencement is associated with a higher rate of ALT elevation in patients with cirrhosis. ${ }^{31}$ However, none of our patients discontinued treatment because the ALT level raised to 5-fold that of the normal upper limit. Of 53 patients with HCC at baseline, 54.7\% (29/53) achieved SVR12. Seven HCC patients ceased treatment because of loss to followup, the development of adverse events, or the absence of any response. Three died of HCC progression. DCV+ASV have been reported to be relatively safe even in patients with compensated cirrhosis and/or HCC.

DCV+ASV can be safely prescribed for patients with mildto-moderate renal impairment; dose adjustments are not necessary. ${ }^{32}$ However, few data are available on whether doses should be adjusted for patients with severe renal impairment/end-stage renal disease. We found that $92.3 \%$ of patients with eGFR $<50$ $\mathrm{mL} / \mathrm{min} / 1.73 \mathrm{~m}^{2}$ achieved SVR12. One patient with chronic kidney disease voluntarily discontinued treatment. 
Current treatment goals for HCV patients focus on viral eradication; however, improvement of fibrosis is also important in patients treated with DAAs. ${ }^{21}$ Thus, serial estimation of fibrosis status is required to predict treatment response and prognosis. Liver biopsy is an imperfect gold standard; being prone to interobservational variation in pathologists' reports; also, the tissue samples obtained may not be representative of the entire liver. Thus, the use of noninvasive markers of fibrosis to follow-up patient treated with DAAs is considered. Previous report has shown that the LS value at the time of SVR usefully predicted liver-related events in CHC patients treated with pegIFN/RBV. ${ }^{33}$ Thus, noninvasive markers should be evaluated and considered to incorporate as surveillance protocols. ${ }^{34}$ However, a reduction of LS value did not always correlated with the improvement of portal hypertension. ${ }^{35}$ Thus, HVPG should be monitored together with the changes of LS value.

Our study had several limitations. First, the study was retrospective, so data collection was not as strict as mandated in clinical trials. However, we collected all data prospectively using a unified in-house protocol. Second, we did not perform liver biopsy (this is the gold standard for assessment of fibrosis regression). It is ethically difficult to perform serial liver biopsies. Thus, we have no pathologic confirmation of our assessments of liver fibrosis. Thus, the exact mechanism such as the improvement of fibrosis or inflammation was hard to be confirmed. However, recent studies showed that LS values compared with FIB4/APRI was fairly reliable to predict fibrosis and treatment response in patients with chronic viral hepatitis. ${ }^{36,37}$ Further long-term follow-up data are needed. Lastly, for patients who have failed DCV+ASV treatment, the following treatment regimen has yet to set guidelines and is waiting for other new drugs.

In conclusion, DCV+ASV treatment was generally well tolerated in real-world clinical practice, with few discontinuations caused by the development of adverse events. Patients with $\mathrm{CHC}$ exhibited virological responses similar to those noted in clinical trials; and safety profile was also similar.

\section{CONFLICTS OF INTEREST}

No potential conflict of interest relevant to this article was reported.

\section{ACKNOWLEDGEMENTS}

This work was supported by a grant from the Korea Health Technology R\&D Project through the Korea Health Industry Development Institute (KHIDI), funded by the Ministry of Health \& Welfare, Republic of Korea (HI15C2859).

The authors are grateful to Ha Kyung Jung (medical record reviewer, Yonsei Liver Center, Yonsei University College of Medicine, Seoul, Korea) for the help with this work.

\section{REFERENCES}

1. Miyaki E, Imamura M, Hiraga N, et al. Daclatasvir and asunaprevir treatment improves liver function parameters and reduces liver fibrosis markers in chronic hepatitis C patients. Hepatol Res 2016;46:758-764.

2. Zhu RX, Seto WK, Lai CL, Yuen MF. Epidemiology of hepatocellular carcinoma in the Asia-Pacific region. Gut Liver 2016;10:332339.

3. Kiyosawa K, Sodeyama T, Tanaka E, et al. Interrelationship of blood transfusion, non-A, non-B hepatitis and hepatocellular carcinoma: analysis by detection of antibody to hepatitis $\mathrm{C}$ virus. Hepatology 1990;12(4 Pt 1):671-675.

4. Niederau C, Lange S, Heintges T, et al. Prognosis of chronic hepatitis C: results of a large, prospective cohort study. Hepatology 1998;28:1687-1695.

5. Gower E, Estes C, Blach S, Razavi-Shearer K, Razavi H. Global epidemiology and genotype distribution of the hepatitis $C$ virus infection. J Hepatol 2014;61(1 Suppl):S45-S57.

6. Kim BK, Jang ES, Kim JH, et al. Current status of and strategies for hepatitis C control in South Korea. Clin Mol Hepatol 2017;23:212218.

7. Gaetano JN. Benefit-risk assessment of new and emerging treatments for hepatitis C: focus on simeprevir and sofosbuvir. Drug Healthc Patient Saf 2014;6:37-45.

8. Kanda T, Imazeki F, Yokosuka 0. New antiviral therapies for chronic hepatitis C. Hepatol Int 2010;4:548-561.

9. Gao M. Antiviral activity and resistance of HCV NS5A replication complex inhibitors. Curr Opin Virol 2013;3:514-520.

10. Cho BW, Kim SB, Song IH, et al. Efficacy and safety of daclatasvir plus asunaprevir for Korean patients with HCV genotype Ib infection: a retrospective multi-institutional study. Clin Mol Hepatol 2017;23:51-56.

11. Kao JH, Jensen DM, Manns MP, et al. Daclatasvir plus asunaprevir for HCV genotype $1 \mathrm{~b}$ infection in patients with or without compensated cirrhosis: a pooled analysis. Liver Int 2016;36:954-962.

12. McPhee F, Sheaffer AK, Friborg J, et al. Preclinical profile and characterization of the hepatitis C virus NS3 protease inhibitor asunaprevir (BMS-650032). Antimicrob Agents Chemother 2012;56:5387-5396.

13. Chayama K, Takahashi S, Toyota J, et al. Dual therapy with the nonstructural protein $5 \mathrm{~A}$ inhibitor, daclatasvir, and the nonstructural protein 3 protease inhibitor, asunaprevir, in hepatitis $\mathrm{C}$ virus genotype 1b-infected null responders. Hepatology 2012;55:742748.

14. Kumada H, Suzuki Y, Ikeda K, et al. Daclatasvir plus asunaprevir for chronic HCV genotype 1b infection. Hepatology 2014;59:2083 2091.

15. Suzuki Y, Ikeda K, Suzuki F, et al. Dual oral therapy with daclatasvir and asunaprevir for patients with HCV genotype $1 \mathrm{~b}$ infection and limited treatment options. J Hepatol 2013;58:655-662.

16. Manns M, Pol S, Jacobson IM, et al. All-oral daclatasvir plus asu- 
naprevir for hepatitis $\mathrm{C}$ virus genotype $1 \mathrm{~b}$ : a multinational, phase 3, multicohort study. Lancet 2014;384:1597-1605.

17. Muir AJ, Poordad F, Lalezari J, et al. Daclatasvir in combination with asunaprevir and beclabuvir for hepatitis C virus genotype 1 infection with compensated cirrhosis. JAMA 2015;313:1736-1744.

18. Asahina Y, Tsuchiya K, Tamaki N, et al. Effect of aging on risk for hepatocellular carcinoma in chronic hepatitis $\mathrm{C}$ virus infection. Hepatology 2010;52:518-527.

19. Nishiguchi S, Shiomi S, Nakatani S, et al. Prevention of hepatocellular carcinoma in patients with chronic active hepatitis C and cirrhosis. Lancet 2001;357:196-197.

20. Morgan RL, Baack B, Smith BD, Yartel A, Pitasi M, Falck-Ytter Y. Eradication of hepatitis $\mathrm{C}$ virus infection and the development of hepatocellular carcinoma: a meta-analysis of observational studies. Ann Intern Med 2013;158(5 Pt 1):329-337.

21. Elsharkawy A, Alem SA, Fouad R, et al. Changes in liver stiffness measurements and fibrosis scores following sofosbuvir based treatment regimens without interferon. J Gastroenterol Hepatol 2017;32:1624-1630.

22. Everson GT, Sims KD, Rodriguez-Torres M, et al. Efficacy of an interferon- and ribavirin-free regimen of daclatasvir, asunaprevir, and BMS-791325 in treatment-naive patients with HCV genotype 1 infection. Gastroenterology 2014;146:420-429.

23. Kwo PY, Cohen SM, Lim JK. ACG clinical guideline: evaluation of abnormal liver chemistries. Am J Gastroenterol 2017;112:18-35.

24. Ziol M, Handra-Luca A, Kettaneh A, et al. Noninvasive assessment of liver fibrosis by measurement of stiffness in patients with chronic hepatitis C. Hepatology 2005;41:48-54.

25. Adler M, Gulbis B, Moreno C, et al. The predictive value of FIB-4 versus FibroTest, APRI, FibroIndex and Forns index to noninvasively estimate fibrosis in hepatitis $\mathrm{C}$ and nonhepatitis $\mathrm{C}$ liver diseases. Hepatology 2008;47:762-763.

26. Sterling RK, Lissen E, Clumeck N, et al. Development of a simple noninvasive index to predict significant fibrosis in patients with HIV/HCV coinfection. Hepatology 2006;43:1317-1325.

27. Petersen JR, Stevenson HL, Kasturi KS, et al. Evaluation of the aspartate aminotransferase/platelet ratio index and enhanced liver fibrosis tests to detect significant fibrosis due to chronic hepatitis C.
J Clin Gastroenterol 2014;48:370-376.

28. Chon YE, Jung ES, Park JY, et al. The accuracy of noninvasive methods in predicting the development of hepatocellular carcinoma and hepatic decompensation in patients with chronic hepatitis B. J Clin Gastroenterol 2012;46:518-525.

29. Seong MH, Kil H, Kim YS, et al. Clinical and epidemiological features of hepatitis C virus infection in South Korea: a prospective, multicenter cohort study. J Med Virol 2013;85:1724-1733.

30. Korean Association for the Study of the Liver (KASL). KASL clinical practice guidelines: management of hepatitis C. Clin Mol Hepatol 2014;20:89-136.

31. Morio K, Imamura M, Kawakami Y, et al. Real-world efficacy and safety of daclatasvir and asunaprevir therapy for hepatitis $\mathrm{C}$ virusinfected cirrhosis patients. J Gastroenterol Hepatol 2017;32:645650.

32. Suda G, Kudo M, Nagasaka A, et al. Efficacy and safety of daclatasvir and asunaprevir combination therapy in chronic hemodialysis patients with chronic hepatitis C. J Gastroenterol 2016;51:733740.

33. Moon C, Jung KS, Kim DY, et al. Lower incidence of hepatocellular carcinoma and cirrhosis in hepatitis $\mathrm{C}$ patients with sustained virological response by pegylated interferon and ribavirin. Dig Dis Sci 2015;60:573-581.

34. Lee HW, Chon YE, Kim SU, et al. Predicting liver-related events using transient elastography in chronic hepatitis $\mathrm{C}$ patients with sustained virological response. Gut Liver 2016;10:429-436.

35. Lens S, Alvarado-Tapias E, Mariño Z, et al. Effects of all-oral antiviral therapy on HVPG and systemic hemodynamics in patients with hepatitis C virus-associated cirrhosis. Gastroenterology 2017;153:1273-1283.e1.

36. Wai CT, Greenson JK, Fontana RJ, et al. A simple noninvasive index can predict both significant fibrosis and cirrhosis in patients with chronic hepatitis C. Hepatology 2003;38:518-526.

37. Tada T, Kumada T, Toyoda H, et al. Improvement of liver stiffness in patients with hepatitis $\mathrm{C}$ virus infection who received directacting antiviral therapy and achieved sustained virological response. J Gastroenterol Hepatol 2017;32:1982-1988. 